\title{
Analysis of the transmission performance of optical signals based on duty cycle division multiplexing (DCDM)
}

\begin{abstract}
In this paper, duty cycle division multiplexing (DCDM) is proposed as an alternative multiplexing technique. It can be applied in any communication systems, although the focus in this paper is in optical fiber communications. In this paper, we examine 3 channels each operating at 10 Gbps modulation rate over a single optical carrier. The performance comparison is made against $30 \mathrm{Gbps} \mathrm{RZ}$ transmitted pulses, time domain multiplexed (TDM). The results show that at a fixed transmission power, DCDM can support longer distance than that with RZ TDM technique. Also, the results show that RZ-TDM pulses require $35.5 \mathrm{~dB}$ more SNR to support the same distance as that for DCDM.
\end{abstract}

Keyword: Multiplexing; Duty cycle; Optical communication 